\title{
UN ESTUDIO DE LA DINAMICA POBLACIONAL DEL RONSOCO O CAPIBARA (Hydrochoerus hidrochaeris) EN EL RIO PACAYA, PERU
}

Pekka Soini N. *

\section{RESUMEN}

Se realizó un estudio a largo plazo de la dinámica poblacional del ronsoco (Hydrochoerus hidrochaeris) en bosque aluvial amazónico, en la Reserva Nacional Pacaya-Samiria. La población estudiada estaba compuesta por familias, parejas y animales solitarios. El tamaño de los grupos variaba de 2 a 15, con un promedio de 4.8 integrantes. El tamaño promedio de los grupos reproductivos fue de $5.5( \pm 2.2$ DE) animales. El tamaño y Composición de estos sufría cambios estacionales debido al influjo estacional de crías a la población. Los grupos se componían de una pareja adulta y su prole de hasta 2 camadas sucesivas. Algunos grupos contenían una segunda hembra adulta. Los animales adultos y subadultos comprendían el $57 \%$ de la población total, los juveniles el $19 \%$ y los infantes el $24 \%$. El ronsoco se reproduce durante todo el año, pero se observó un pico anual de nacimientos en agostosetiembre, en el que el $81 \%$ de los grupos tenía cría. Las camadas consistían en 1 a $6(\mathrm{x}=3.8)$ crías y los intervalos registrados entre sucesivas camadas variaban de 5 $1 / 2$ a 11 meses. Aproximadamente el $40 \%$ de las crías sobrevivió hasta el segundo año de vida. Generalmente sólo 1 ó 2 (o ninguna) crías por carnada sobrevivieron hasta la etapa subadulta y se estima que no más del $20 \%$ de los animales nacidos en la población llega a reproducir. Las crecientes grandes del río, enfermedades, predación y agresión intraespecítica fueron las principales causas de mortalidad en la población.

\section{SUMMARY}

Population dynamics of the capybara (Hydrochoerus hydrochaeris) was studidied in Amazonian floodplain forest, in the Pacaya - Samiria National Reserve, over several years. The study population was compposed of family groups, pairs, and solitary animals. Group size varied from $2-15(\mathrm{x}=4.8)$; the mean size for reproductive groups was $5.5+2.2$ S.D. animals. The groups size and composition showed seasonal changes, due to seasonal requirement of infants into the population.

* Investigador IIAP. Av. Abelardo Quiñones Km. 2.5. Apartado 784. Iquitos-Perú. 
The groups were composed of one adult pair and their offspring of up to 2 successive litters. Sorne groups contained and additional adult female. Adult and subadult animals made up $57 \%$ of the total population, juveniles $19 \%$, and infants $24 \%$. Reproduction took place year-round, hut a brith peak was evident in August September during wich $81 \%$ or the groups had young. The litters consisted of 1-6 (x $=3.8$ ) young, and recorded interbirt intervals ranged from $51 / 2-11$ months. About $40 \%$ of the young survived to beyond the first year of life. Generally only 1 or 2 (or none) young per litter reached subadult age class, and it is estimated that no more than $20 \%$ of the offspring survive to reproduce.

Excepcionally high floods, disease, predation, and intraspecific aggression were the principal causes of mortality in the study population.

Palabras claves: Hydrochoerus hydrochaeris, dinámica poblacional, estructura poblacional, reproducción, mortalidad, organización social, Reserva Nacional Pacaya-Samiria.

\section{INTRODUCCIÓN}

El ronsoco, Hydrochoerus hydrochaeris, es el roedor conocido de mayor tamaño. De costumbres semiacuáticas, habita los humedales tropicales, incluyendo las sabanas inundables, bosques de ribera y pantanales, desde Venezuela hasta Argentina septentrional (Ojasti, 1973; Mones y Ojasti, 1986).

Es un animal corpulento, alcanza una longitud mayor de un metro y altura de medio metro, con un peso máximo registrado de $91 \mathrm{Kg}$. (Mones y Qjasti, 1986). Una hembra adulta cazada en Puerto Perú, Río Ucayali el 15 de abril de 1978, y que examinamos tenía una longitud total de $125 \mathrm{~cm}$. y peso de $60 \mathrm{Kg}$. Tres machos adultos cazados en el Río Tahuayo en julio de 1986 pesaron 34, 55 y $45 \mathrm{Kg}$. respectivamente (R. bodmer, comunicación personal). El tamaño de los adultos varía considerablemente y no parece existir diformismo sexual en cuanto al tamaño (Ojasti, 1973; Sehaller y Crawshaw, 1981). En ambos sexos los genitales permanecen ocultos en un saco cutáneo anal, lo que dificulta la identificación del sexo en el campo. No obstante, los machos adultos son reconocibles por la presencia de una conspicua glándula cebácea sobre el hocico.

Debido a su gran potencialidad para manejo con fines de aprovechamiento de su carne y cueros, la dinámica poblacional del ronsoco ha sido objeto de algunos estudios intensivos en los llanos de Venezuela (Ojasti, 1973; Macdonald, 1981) y Colombia Jorgenson, 1986) y en el Pantanal de Matto Grosso, brasil (Schaller y Crawshaw 1981). Sin embargo, no existe información adecuada sobre la dinámica poblacional de la especie en el bosque lluvioso de la Amazonía. 
En cumplimiento del programa de investigación y manejo de la fauna de la Reserva Nacional Pacaya-Samiria, Perú, se ha estado realizando, a partir del año 1979, un estudio de largo plazo de la ecología, dinámica poblacional y comportamiento del ronsoco en la cuenca del río Pacaya. En este artículo presentamos los resultados en cuanto a la dinámica poblacional de la especie se refiere; los aspectos de la ecología y densidades poblaciones ya fueron tratados en trabajos anteriores (Soini, 1992; Soini y Soini, 1993).

Para los fines del presente estudio, los animales fueron clasificados en 3 categorías o niveles de edad, definidos como sigue:

INFANTES : Desde nacimiento hasta 4 meses de edad (según Ojasti (1973) y Zara (1973) son amamantados hasta aproximadamente 4 meses). Tamaño pequeño (menos de la mitad del tamaño adulto); longitud de la pisada del pie trasero menor de $9 \mathrm{~cm}$.

JUVENILES : Desde 4 meses hasta cumplir 1 año. Tamaño conspicuamente menor que el de los adultos; longitud de la pisada del pie trasero $9-11 \mathrm{~cm}$.

ADULTOS Y : Edad 1 año o más. Longitud de la pisada del pie, trasero mayor SUBADULTOS de $10 \mathrm{~cm}$.

En este estudio no siempre fue posible discriminar entre los adultos y subadultos, por lo que las dos categorías fueron combinadas. El tamaño Corporal de los subadultos grandes se traslapa ampliamente Con el de los adultos pequeños, lo que muchas veces dificultó la discriminación entre un animal subadulto y una hembra adulta, ya que ambos carecen de la conspicua glándula nasal. Según Zara (1973) y Ojasti (1973) respectivamente, ambos sexos alcanzan la madurez sexual a los 15 meses o $1 \frac{1}{2}$ años de edad.

Al observar un grupo (o individuo) de ronsocos anotamos datos sobre la ubicación, actividad, comportamiento, tamaño y composición del grupo. Además de la observación visual directa, el tamaño de las huellas de las pisadas se utilizó como indicador auxiliar al asignar los integrantes de un grupo a las categorías de edad.

\section{MÉTODOS}

El estudio se realizó primariamente en el área de la Estación Biológica de Cahuana, abarcando los terrenos colindantes con un tramo de $3 \mathrm{kms}$. del río y con la 
tipishca Cahuana; y secundariamente con un tramo de $12 \mathrm{kms}$ del río, desde Cahuana de surcada hasta $2 \mathrm{kms}$ más arriba de la cocha Mauca Tapaje (gráfico 1). Una descripción del área de estudio y la del hábitat del ronsoco las dio Soini y Soini (1993).

En el área de Cahuana se realizaron censos de los grupos de ronsocos y observaciones ecológicas intermitentemente durante todos los años desde 1979 hasta 1988. En el tramo Cahuana-Mauca esto se hizo principalmente en el período de 1982-1984. Aunque las observaciones abarcan todos los meses del año, el contacto con los grupos fue siempre más frecuente durante la segunda mitad del año, particularmente en el período setiembre-noviembre, cuando las orillas del río no se encuentran inundadas; durante la temporada anual de inundación fue siempre más difícil ubicar y observar los ronsocos.

Las observaciones se realizaron de día y de noche. En nuestra área de estudio los ronsocos fueron más activos y/o observables de noche que de día, particularmente durante las horas anteriores a la medianoche y en las de la madrugada, hasta el amanecer, por lo que la mayoría de las observaciones fueron llevadas a cabo en aquellas horas. Durante el día tendían a permanecer dentro de la espesura de la vegetación, mientras que de noche (y hasta las horas del amanecer), salían a menudo a las playas y otras orillas abiertas, donde las condiciones de observación eran mejores. Las observaciones nocturnas se realizaron a la luz de la luna y/o con ayuda de linternas.

De noche los ronsocos fueron generalmente ubicados y observados desde una canoa; de día fueron observados desde canoa o escondidos en la vegetación de la orilla opuesta. El grupo principal de estudio, que vivía en el entorno de la estación biológica y estaba más habituado a nuestra presencia, muchas veces pudo ser observado de muy cerca y de noche inclusive desde el interior de la casa.

\section{RESULTADOS Y DISCUSION}

\section{TAMAÑO Y COMPOSICIÓN DE LAS UNIDADES SOCIALES}

Las unidades sociales observadas en la población de estudio comprendían animales solitarios, parejas y grupos familiares. El tamaño de los grupos variaba de 2 a 15 animales, con un promedio de $4.8 \pm 2.4$ DE.. ( $n=120$ grupos). La muestra está basada en censos mensuales de los grupos presentes en el área de estudio dentro del período 1979-1988 (cuadro 1). Para cada mes de censo, sólo se incluyó los grupos observados bajo buenas condiciones de observación, que permitían un conteo aparentemente completo de los integrantes del grupo. Para cada grupo, observado repetidamente dentro del mismo mes, se consideró para el censo el máximo número de integrantes alcanzado en el grupo durante ese mes. 
El gráfico 2 muestra la distribución de frecuencias de tamaño de los grupos. El tamaño modal fue de 2 animales. Estos últimos grupos consistían mayormente en parejas reproductivas; es decir, los que estaban criando o habían criado; el tamaño modal fue de 5 animales y el tamaño promedio $5.5 \pm 2.2 \mathrm{DE}(\mathrm{n}=94$ grupos).

La composición promedio por niveles de edad de los grupos de la muestra total ( $\mathrm{n}=20$ grupos) fue la siguiente:

$$
\text { X D.E. (rango) }
$$

Adultos +

$2.4 \pm 1.2$

subadultos

Juveniles

$$
1.8 \pm 0.7
$$

Infantes

$$
2.7 \pm 1.5
$$

Una examen más detallado de la composición de los grupos reproductivos indicó que éstos sufrían cambios estacionales causados principalmente por el influjo marcadamente estacional de infantes a la población (ver "Reproducción") y de la maduración y (mortalidad) subsiguiente de éstos. La composición promedia bimensual de los grupos censados (cuadro 2) refleja bien estos cambios. 


\section{Cuadro 1}

RESULTADOS DE LOS CENSOS MENSUALES DE LA COMPOSICIÓN DE LOS GRUPOS DE RONSOCOS EN EL AREA DE ESTUDIO EN EL PERIODO 1979-1988.

\begin{tabular}{|c|c|c|c|c|c|c|c|c|}
\hline \multicolumn{2}{|c|}{$A+S A J$} & $\mathrm{I}$ & \multicolumn{2}{|c|}{$\mathrm{A}+\mathrm{SAJ}$} & I & \multicolumn{2}{|c|}{$\mathrm{A}+\mathrm{SA} \mathrm{J}$} & I \\
\hline \multicolumn{3}{|c|}{ ENERO } & \multicolumn{3}{|c|}{ AGOSTO } & \multicolumn{3}{|c|}{ NOVIEMBRE } \\
\hline 2 & - & - & 4 & 3 & - & 2 & 3 & \\
\hline 2 & - & - & 2 & - & - & 2 & 1 & 4 \\
\hline 2 & 2 & 1 & 2 & - & - & 2 & - & - \\
\hline 2 & 3 & - & 2 & - & - & 2 & 2 & 1 \\
\hline 3 & 2 & - & 2 & - & - & 2 & - & 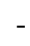 \\
\hline 2 & 2 & 5 & 2 & - & - & 2 & - & 3 \\
\hline \multirow[t]{3}{*}{1} & 2 & 2 & 2 & - & - & 2 & - & 0 \\
\hline & & & 2 & 2 & 2 & 2 & 1 &  \\
\hline & & & 2 & - & 1 & 2 & 1 & \\
\hline
\end{tabular}

\begin{tabular}{llllll}
\multicolumn{2}{l}{ FEBRERO } & & \multicolumn{4}{l}{ SETIEMBRE } \\
3 & 2 & - & 3 & 3 & 1 \\
2 & 2 & - & 2 & - & 6 \\
2 & - & - & 2 & - & - \\
2 & - & - & 2 & 2 & 1 \\
2 & 3 & - & 2 & - & - \\
5 & 1 & 2 & 2 & - & 5 \\
2 & 2 & - & 2 & - & 5 \\
& & & 2 & 2 & - \\
& & & 2 & 2 & 3 \\
MARZO & & 2 & 2 & - \\
2 & - & - & 2 & - & 4 \\
2 & 2 & - & 2 & - & - \\
2 & 1 & - & 2 & - & 2 \\
2 & 1 & - & 2 & - & - \\
2 & 1 & 4 & 2 & - & 1 \\
5 & 1 & 2 & 2 & - & 3 \\
& & & 2 & - & 1 \\
ABRIL & & & 6 & 2 & 6 \\
2 & 3 & - & 8 & 1 & 6 \\
2 & - & 5 & 3 & 3 & - \\
2 & 2 & - & 2 & 2 & 2 \\
2 & 1 & - & 3 & 3 & -
\end{tabular}




$\begin{array}{llllll}2 & 1 & - & 2 & - & - \\ 4 & 1 & 2 & 3 & 2 & 5 \\ & & & 2 & - & -\end{array}$

Continua Cuadro 1.

$\begin{array}{llllll}\text { MAYO } & & & 2 & - & 3 \\ 2 & 1 & 4 & 2 & - & 2 \\ 2 & 2 & 4 & 4 & 1 & 1 \\ 2 & - & - & 2 & - & 4 \\ 4 & 3 & - & 2 & 2 & - \\ 4 & 1 & 2 & 2 & - & 1 \\ & & & 4 & 2 & - \\ \text { JUNIO } & & & 4 & 1 & 2 \\ & & & 2 & 2 & 1 \\ 2 & - & - & 2 & - & - \\ 2 & - & 1 & & & \\ 2 & 2 & - & \text { OCTUBRE } \\ 4 & 3 & - & & & \\ 3 & 1 & 2 & 2 & 3 & 1 \\ & & & 3 & 2 & 5\end{array}$

$\begin{array}{llllll}\text { JULIO } & & & 2 & - & - \\ 2 & 1 & - & 2 & 2 & 1 \\ 2 & - & - & 2 & - & 3 \\ 3 & - & - & 2 & 2 & 3 \\ 2 & 2 & - & 2 & 2 & - \\ 4 & 3 & - & 2 & - & 3 \\ 2 & 1 & 2 & 2 & - & - \\ & & & 2 & - & - \\ & & & 2 & 2 & -\end{array}$

$\mathrm{A}+\mathrm{AS}=$ Adultos + subadultos $; \quad \mathrm{J}=$ Juveniles $; \quad \mathrm{I}=$ Infante 


\section{Cuadro 2}

COMPOSICIÓN PROMEDIO BIMENSUAL DE LOS GRUPOS
REPRODUCTIVOS DE RONSOCOS EN EL AREA DE ESTUDIOS

Set. oct. Nov.dic. Ene-feb. Mar-abr. May-jun. Jul.ago.

$\begin{array}{llllll}\text { Inf. } 2.2 & 1.7 & 1.4 & 1.2 & 1.1 & 0.6 \\ \text { Juv. 1.12 } & 1.1 & 1.9 & 1.3 & 1.6 & 1.5 \\ \text { A + Sa 2.6 } & 2.0 & 2.4 & 2.4 & 2.9 & 2.6\end{array}$

En setiembre-octubre, cuando la gran mayoría de los grupos esta con nuevas camadas de crías, el número de infantes presentes en la población alcanza su máximo nivel; de allí disminuye paulatinamente, por mortalidad y maduración, hasta el inicio de esa temporada. La reducción del número de adultos + subadultos en noviembrediciembre parece deberse a una mayor incidencia de dispersión de los subadultos en esa temporada.

Se observó animales solitarios en todas las temporadas. Es probable que la frecuencia de éstos sufriera alguna fluctuación estacional, pero esto no se pudo verificar con datos cuantitativos, ya que muchas veces los solitarios no eran individualmente reconocibles. Lo mismo podría decirse de las parejas incipientes.

\section{PROPORCIÓN MACHOS/HEMBRAS}

Por regla general cada grupo tenía sólo un macho adulto y una hembra adulta, pero algunos grupos parecían tener temporalmente hasta 2 hembras adultas. En algunos casos, y posiblemente en todos, se trataba de una hembra reproductiva acompañada por su hija ya madura. Exceptuando los dos grupos más grandes observado, en cada grupo sólo había una hembra reproductiva activa. Los dos grupos vistos cada uno en sólo una oportunidad; tenían, respectivamente, 6 y 5 adultos/subadultos más juveniles o infantes, pero no fue posible identificarlos por sexo, y posiblemente hubo dos hembras reproductivas en estos grupos. En conclusión, aunque moralmente los grupos contenían sólo un adultos de ambos sexos, en termino medio los grupos contenían un número ligeramente mayor de hembras que machos. Entre los solitarios observamos animales de ambos sexos, pero no fue posible determinar la proporción entre ellos. 


\section{ESTRUCTURA POBLACIONAL}

La composición porcentual por niveles de edad de la población contenida en los grupos censados fue la siguiente:

$\begin{array}{ll}\text { Adultos + subadultos } & 50.1 \% \\ \text { Juveniles } & 22.2 \% \\ \text { Infantes } & 27.7 \%\end{array}$

Para estimar la estructura de la población total debemos considerar también los animales solitarios presentes en la población (todos ellos eran subadultos o adultos). En base a los censos poblacionales realizados en el tramo Cahuana-Mauca en 1982-1984 (tratado más detalladamente en Soini, 1992 y en prep.), estimamos que los solitarios comprenden, en término medio, el $23 \%$ de la población adulta/subadulta.

Los censos fueron realizados en agosto-setiembre; es posible que la proporción de solitarios sufra alguna fluctuación estacional, pero para el presente cómputo consideramos este porcentaje como representativo de todo el año. Agregando este componente a la categoría de adultos + subadultos, la población del área de estudio tendría la siguiente estructura:

$\begin{array}{ll}\text { Adultos + subadultos } & 57 \% \\ \text { Juveniles } & 19 \% \\ \text { Infantes } & 24 \%\end{array}$

\section{REPRODUCCIÓN}

El ciclo de del ronsoco es espontáneo y tiene una duración promedia de 7.5 días (López-Barbella, 1982). La gestación dura aproximadamente 5 meses (Donaldon et al; 1975) Mones y Qjasti, 1986; Zara 1973). A partir de 15-21 días después del parto la hembra puede entrar nuevamente en estro (Azcárate, 1976).

\section{DISTRIBUCIÓN TEMPORAL DE LOS NACIMIENTOS}

Para 29 camadas de crías nacidas en el transcurso del estudio pudimos constatar la fecha aproximada o el mes de nacimiento. La distribución temporal de éstos (gráfico 3) indica que en el Río Pacaya los ronsocos se reproducen durante todo el año. Sin embargo, un pico de nacimientos bien definido es evidente en el 
período agosto- setiembre; definido es evidente en el período agosto-setiembre; nuestros registros de los años de más intensivo seguimiento (el período 1979-1981 en el área de Cahuana y 1982-1984 en el de Cahuana-Mauca) indican que, anualmente, en término medio, el $81 \%$ de los grupos de la población (excluyendo las parejas incipientes) cría en esa temporada.

No hemos observado nacimientos en los dos meses subsiguientes (octubrenoviembre), mientras que a lo largo de todo el período diciembre-junio se observa algunos nacimientos dispersos.

La temporada principal de nacimientos y cría coincide con el período anual de merma del río (estación seca y estación inicial de lluvia), que es la temporada de máxima abundancia de gramalote nuevo (Echinocloa polyatachya y Paspalum sp.) y de otras plantas herbáceas (particularmente Ludwigia spp) preferidas por los ronsocos para la alimentación. Como es sabido para los mamíferos en general, la lactancia es, en términos de energía, el período más exigente dentro del ciclo reproductivo de la hembra (Millars, 1976), por lo que ésta tiende a estar sincronizada con la temporada anual de máxima abundancia de los recursos alimentarios. Además, a parte de la leche materna, las crías de ronsoco ya empiezan a alimentarse de la vegetación en la primera semana de vida; la sincronización de la cría con el período anual de máxima disponibilidad de hierbas tiernas indudablemente contribuye, a mayor supervivencia de las crías nacidas en esta temporada.

Las crías que nacen en la temporada de inundación, o sea las camadas de diciembre-junio, se encuentran evidentemente en desventaja en este sentido. La relativamente baja incidencia de camadas observadas en este último período es probablemente el resultado combinado de dos factores, la disminución de nacimiento y el aumento de mortalidad infantil.

\section{TAMAÑO DE LAS CAMADAS}

En las 21 camadas en que el número exacto de crías presentes puedo ser constatado dentro de los primeros días o semanas de nacidos, el número de crías variaba de 1 a 6 , con un valor promedio de 3.8 ( \pm 1.8 D.E.). El tamaño modal fue de 5 crías, y es probable que el tamaño promedio de las camadas recién nacidas sea en realidad un poco mayor a 3.8., ya que algunas de las camadas incluidas pueden haber sufrido alguna mortalidad perinatal antes de ser observadas por nosotros. En las poblaciones de los llanos de Venezuela se ha observado camadas de hasta 7 crías (Ojasti, 1973), pero en el Pacaya nunca vimos camadas mayores de 6. 


\section{INTERVALOS ENTRE NACIMIENTOS}

La hembra de un grupo, seguido desde marzo de 1982 hasta abril de 1983, tuvo 2 camadas en ese período, con un intervalo de 11 meses. Otro grupo, seguido desde noviembre de 1985 hasta enero de 1988 tuvo 4 camadas en ese período, con intervalos de 8 1/2, 5 1/2 y 9 1/2 meses. Por lo tanto, los intervalos observados en este estudio indican que en el Pacaya las hembras pueden tener hasta 2 camadas por año, pero por regla general los intervalos tienden a ser mayores de 6 meses, aunque menores de 12 meses.

\section{MORTALIDAD}

Nuestras observaciones sobre los tamaños inicial y subsiguiente de las camadas de crías indican que la mortalidad infantil es alta. Esto es evidente cuando comparamos el tamaño promedio inicial de las camadas con el promedio general de las camadas infantiles y con el promedio de las camadas juveniles, a saber:

Tamaño inicial

(edad menor de 1 mes)

Tamaño de infantes en general (edad 0-4 meses)

Tamaño de juveniles (edad 5-12 meses)

$$
3.8 \text { (rango: } 1-6) \quad(\mathrm{n}=21)
$$

2.7 (rango: $1-6) \quad(\mathrm{n}=60)$

1.8 (rango: $1-3) \quad(\mathrm{n}=71)$

Para la etapa juvenil el tamaño modal de las camadas se bahía reducido de 5 a 2 crías y el tamaño máximo de 6 a 3 .

Tomando en cuenta que el tamaño promedio inicial es en realidad probablemente un poco mayor de 3.8 (ver sección tamaño de las camadas) y el hecho de que algunas camadas perecen totalmente en la etapa infantil, podemos estimar que la mortalidad de crías en el primer año de vida es aproximadamente el $60 \%$. Los resultados de un seguimiento más sostenido de desarrollo de 8 camadas (cuadro 3 ) sugieren que la mortalidad es más elevada durante los 2 primeros meses de vida que durante los meses subsiguientes de la etapa infantil/juvenil. 
Nuestras observaciones indican que, por regla general, sólo 1 ó 2, ó a veces ninguna, de las crías originales de una carnada sobreviven hasta la etapa subadulta. Corno subadultos ya desligados de sus grupos natales, viven un período más o menos prolongado de existencia solitaria; y probablemente la mortalidad es relativamente elevada también en ese período, por lo que estimamos que no más del $20 \%$ de los animales nacidos en la población llega a reproducirse.

Según hemos podido observar, los grupos tienen una existencia relativamente breve, generalmente sólo 1-3 años. La muerte de uno de los adultos del grupo parece ser la causa más frecuente de la dispersión de un grupo. Por consiguiente, nuestras observaciones concuerdan con la conclusión de Schaller y Crawshaw (1981) en el sentido que también entre los adultos la tasa de mortalidad es alta, siendo pocos los individuos que sobreviven hasta los 4 años de edad.

\section{CAUSAS DE MORTALIDAD}

Enfermedades, predación y agresión intraespecie son evidentemente causas importantes de mortalidad en la población de estudio. También la excesiva inundación de los terrenos por crecientes excepcionalmente grandes del río, también es un causante importante, e indirecta de mortalidad.

La predación, evidentemente, es la causa principal de mortalidad entre los infantes y juveniles. Los principales predadores de éstos son el "tigre" o jaguar (Panthera onca), la "boa" o anaconda (Eunectes murinus), y los "lagartos" o caimanes (Caiman crocodilus y Melanochus níger). En una oportunidad vimos un jaguar cazar uno de los infantes de nuestro grupo principal de estudio y en otra una anaconda llevarse un subadulto del mismo grupo. Otro predador potencial es el puma (Felis concolor); pero, en contraste con el jaguar, éste parece ser poco abundante en el Pacaya y casi nunca se lo ve en los terrenos de ribera habitada por el ronsoco.

La agresión que a veces culmina en pelea es relativamente frecuente entre los machos de esta especie (Macdonald, 1981; Schaller y Crawshaw, 1981). En todos los anos del estudio vimos machos adultos y subadultos (solitarios) con heridas aparentemente resultantes de agresión intraespecie; y un macho adulto encontrado muerto en nuestra área de estudio evidentemente murió como consecuencia de tales heridas recibidas.

Según hemos podido observar en el transcurso de los años de nuestra estadía en el Pacaya, las crecientes excepcionalmente grandes del río son un factor importante de mortalidad para los mamíferos terrestres del área, dando origen a fluctuaciones periódicas de la abundancia de éstos. El ronsoco, por sus hábitos 
semiacuáticos, es menos afectado que algunos otros mamíferos terrestres, pero tampoco está de todo intocado por tales crecientes. En abril de 1986 la creciente del río Pacaya (y de Ucayali) alcanzó el máximo nivel conocido hasta ahora en la región. Como consecuencia de ello, hasta los terrenos mas elevados quedaron inundados. En nuestra área de estudio hubo una drástica disminución de la población de ronsocos a partir del año 1986. Así por ejemplo la población de Cahuana, que oscilaba alrededor de 30 animales en el período 1979-1984, se redujo en agostosetiembre de 1988, a sólo $8 \pm 2$ animales. Esta declinación fue evidentemente en parte una consecuencia directa de la creciente, por escasez de recursos alimentarios y de lugares secos donde descansar.

Las observaciones de Qjasti $(1971,1973)$ en Venezuela y de Schaller y sus asociados $(1978,1981)$ en Brasil indican que las epidemias de tripanosomiasis equina causado por el protozoario Trypanosoma sp., es importante causante de mortalidad en las poblaciones naturales de ronsocos. Los síntomas más notables son la apatía, emaciación, debilitamiento y pérdida de coordinación de las extremidades posteriores (Ojasti, 1973; Schaller y Vasconcelos, 1978; Schaller y Crawshaw, 1981; Mones y Ojasti 1986).

En diciembre de 1987 la hembra reproductiva de nuestro grupo principal de estudio mostraba los característicos síntomas de tripanosomiasis, incluyendo un progresivo debilitamiento y pérdida de funcionamiento de las extremidades posteriores; pasaba las noches arrastrándose por la hierba y comiéndola echada. Continuó en estas condiciones físicas por un período de 5 semanas y murió el 29/30 de enero de 1988. El macho adulto del grupo había desaparecido ya algo antes, posiblemente a causa de la misma enfermedad. De un total de 7 animales, que contenía el grupo en diciembre, aparentemente sobrevivió sólo un macho juvenil, que sigue viviendo solo en el entorno de la estación biológica.

Los datos de Ojasti $(1971,1973)$ y Schaller \& Vasconcellos (1978) indican que el ataque de una epidemia de tripanosomiasis en una población de ronsocos tiende asociarse con un período ecológicamente crítico o adverso (período de "stress"), cuando los animales se encuentran extenuados y así menos resistentes a enfermedades. Por consiguiente, los datos disponibles parecen indicar que, a parte del efecto inmediato de la creciente, la drástica reducción de la población de ronsocos en el área de estudio, y aparentemente en toda la cuenca del Pacaya, se debería a una epidemia de tripanosomiasis que sobrevino como una consecuencia de la creciente del 1986. 


\section{ORGANIZACIÓN SOCIAL}

Los datos presentados en las secciones anteriores y nuestras observaciones adicionales sobre el comportamiento social de la especie, nos permite hacer algunas conclusiones sobre la organización social del ronsoco en el río Pacaya. La población está constituida por animales solitarios, parejas y grupos familiares formados por una pareja reproductiva y su prole de 1 a 3 camadas consecutivas. Normalmente el tamaño de estos grupos no pasa de 11 animales. En contraste con las poblaciones observadas en hábitat abiertos (Ojasti, 1971, 1973; Macdonald, 1981; Schaller y Crawshaw 1981; Jorgenson, 1986), no se observa grandes manadas con varios adultos de ambos sexos, y sólo raras veces hay hasta 2 hembras: adultas en un grupo.

El hecho de que nunca hemos observado más de un macho adulto por grupo podría indicar que los machos tienden a emigrar o ser expulsados de sus grupos natales en una edad algo más temprana que las hembras. Con la posible excepción de algunas hembras, los animales subadultos emigran de sus grupos natales en el segundo año de vida; luego llevan una vida solitaria hasta formar pareja (o hasta su muerte) con un subadulto/adulto del sexo opuesto, originándose así los nuevos grupos familiares.

\section{BIBLIOGRAFIA}

AZCARATE, 1. 1976. "Algunos datos sobre el comportamiento social en una manada de chigüires (Hyrochoerus hydrochaeris)". II Seminario sobre Chigüires y Babas. Programa y Resúmenes. CONICIT. Venezuela.

DONALSON, S.L., Wirtz, T.B., Hito, A.E. 1975. "The social behavior of capybaras. Hyddrochoerus Hydrochaeris, at Evansville Zoo". Int. Zoo Yb. 15: 201-206.

JORGENSON, J.P., 1986. "Notes on et ecology and behavior of capybaras in northeastern Colombia". en Vida Silv. Neotrop., 1: 31-40.

LÓPEZ-BARBELLA 5., 1982. "Una contribución al estudio de la fisiología reproductiva del chigüire (Hydrochoerus hydrochaeris) en cautiverio. 1 Ciclo estral". en Acta Cient. Venezol., 33: 487-50 1.

MACDONALD, D.W. 1981. "Dwindling resources and the social behavior of capybara (Hydrochoerus hydrochaeris) J. Zool”.,194: 371-392. 
MILLARS, J.S. 1976. "Adaptive features of mammalian reproduction. Evolution”, 31: 370-386.

MONES, A. y Qjasti. J. 1986. Hydrochoerus hydrochaeris. Mammalian Species, $\mathrm{N}^{\mathrm{o}}$ $264: 1-7$.

OJASTI, 3., 1971. "El chigüire", en Defensa de la naturaleza, 1 : 1- 10.

OJASTI, J., 1973. Estudio biológico del chigüire o capibara. Fondo Nac. Investi. Agropec., Caracas, 275 pp.

SCHALLER, G.B. y Crawshaw, P.G. 1981. "Social organization of a capybara population”, en Saugetierkund. Mitt., 29 3-16.

SCHALLER, G.B. y Vasconcellos, J.M.C., 1978. "Jaguar predation on capybara”, en Z. Saugeuterkund., 43:296-301.

SOINI P. 1992. "Densidades poblacionales del ronsoco o capibara (Hydrochoerus hydrochaeris) y el desarrollo de un método de censo". Informe de Pacaya $\mathrm{N}^{\mathrm{o}}$ 34, IIAP/COREPASA, Iquitos. $14 \mathrm{pp}$.

SOINI, P. Y SOINI M. 1993. "Ecología del ronsoco o capibara (Hydrochoerus hydrochaeris) en el río Pacaya-Perú”, en Folia Amazónica.

ZARA, J.L., 1973. "Breeding and husbandry of the capybara, Hydrochoerus hydrochaeris, at Evansille Zoo". Int. Zoo. Yb, . 13:137-139. 


\section{Gráfico 1}

EL AREA DE ESTUDIO EN EL RIO PACAYA

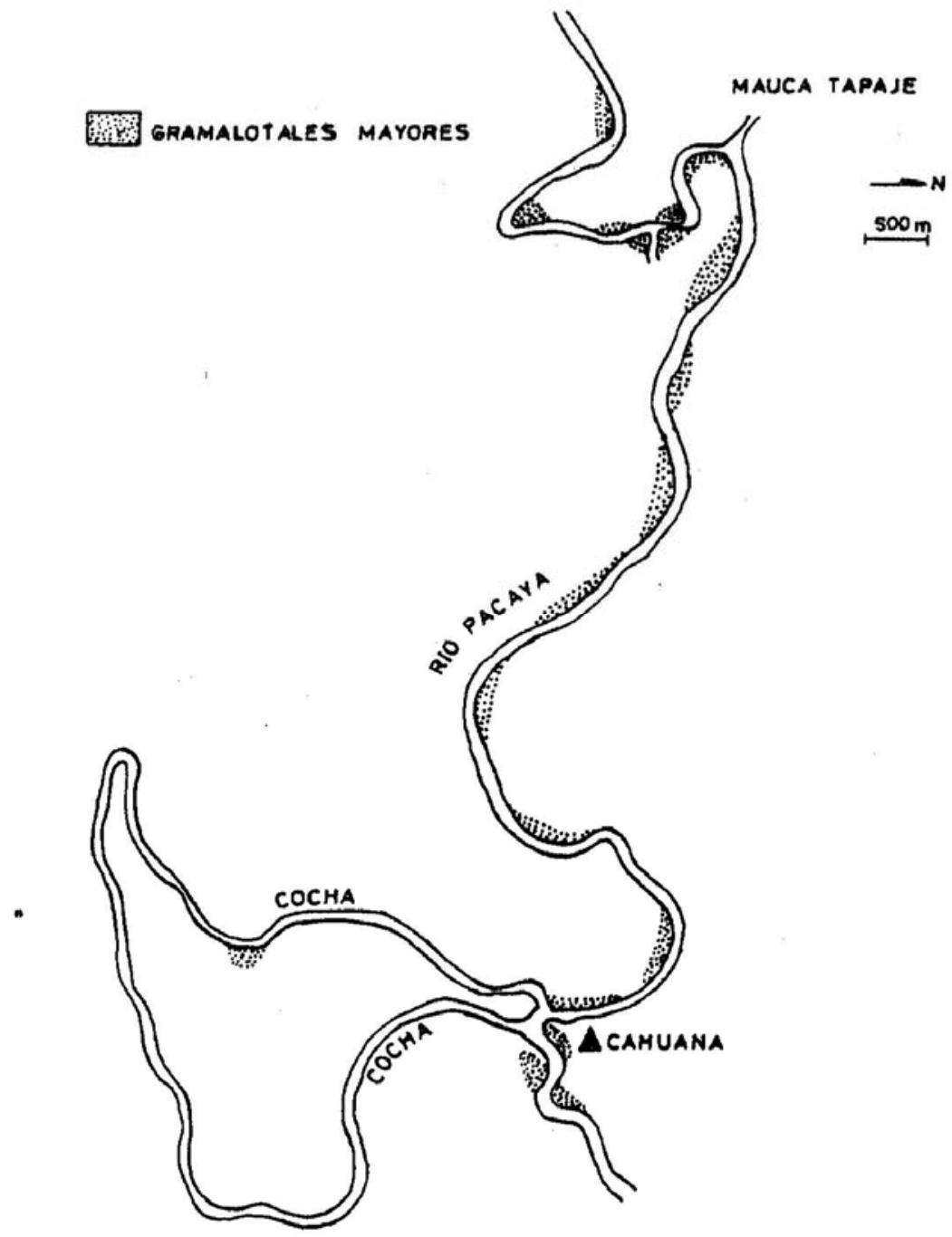




\section{Gráfico 2}

DISTRIBUCION DE FRECUENCIAS DE TAMAÑO DE LOS GRUPOS DE RONSOCOS

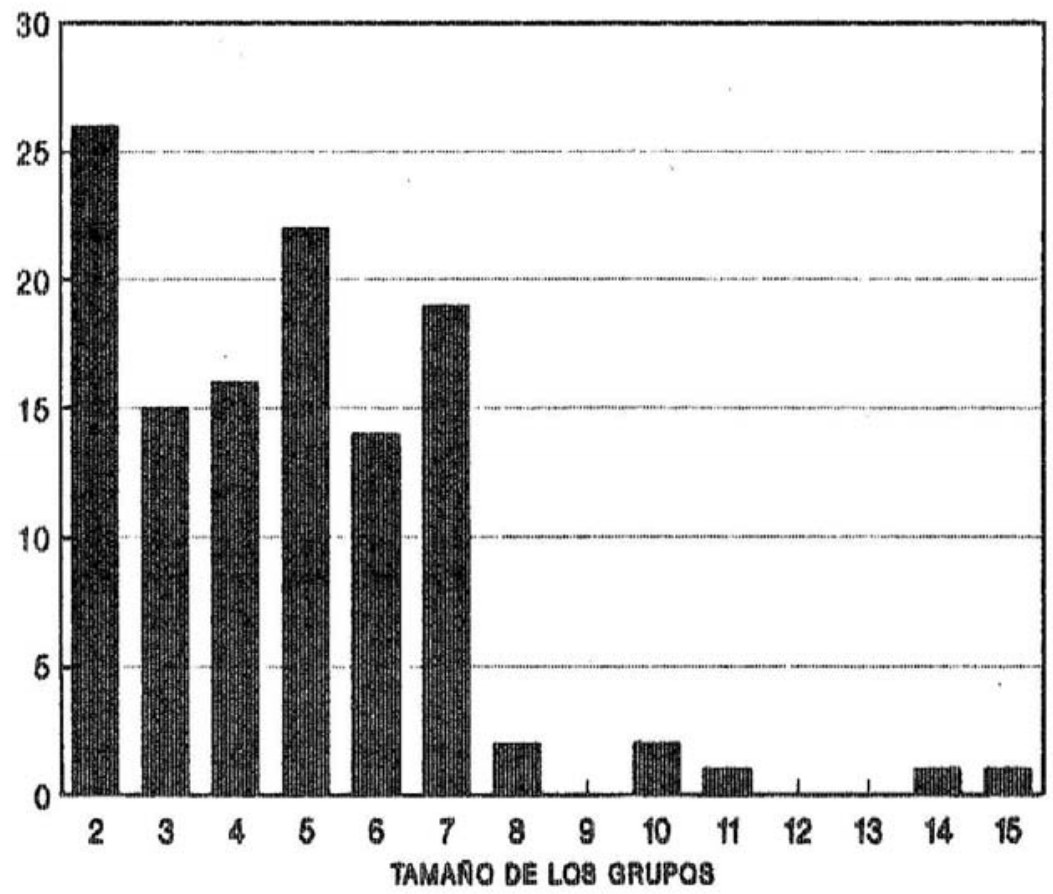




\section{Gráfico 3}

DISTRIBUCION MENSUAL DE LOS NACIMIENTOS EN LA POBLACION DE ESTUDIO

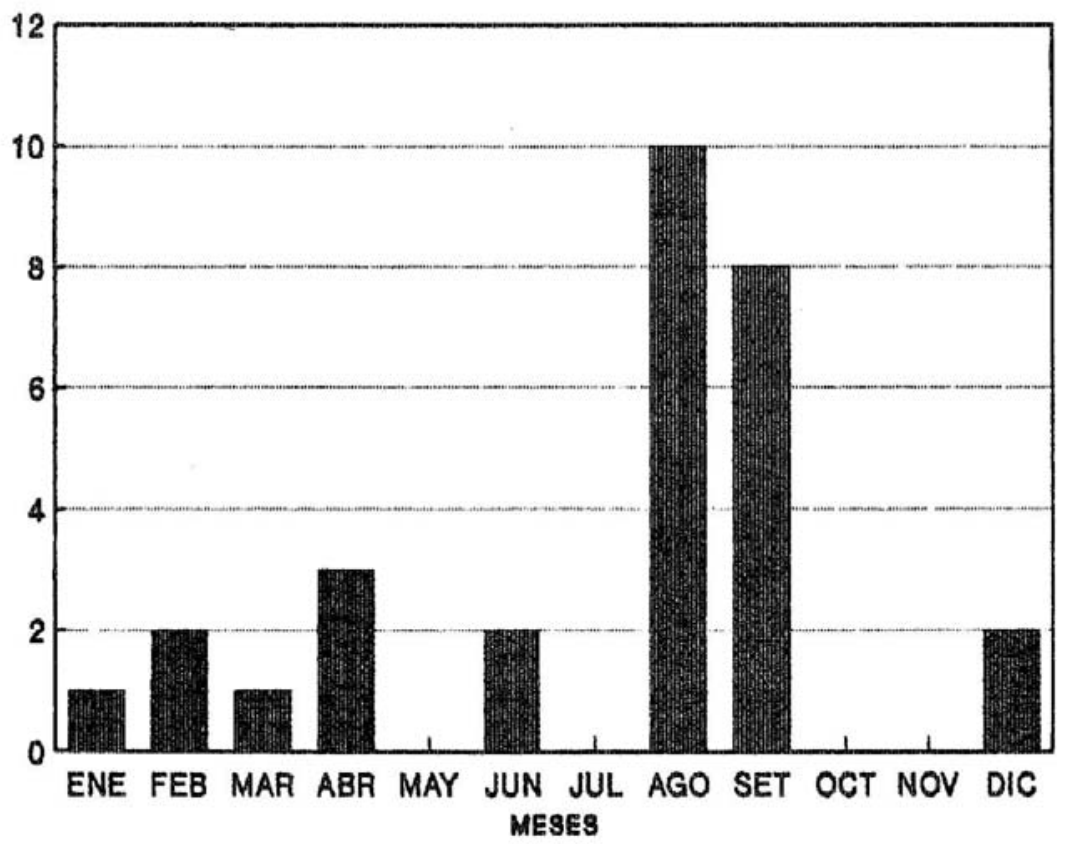

